\title{
TRANSFORMATIONS OF A SURFACE BEARING A FAMILY OF ASYMPTOTIC CURVES*
}

\author{
BX \\ G. D. GORE
}

Introduction. It is the purpose of this paper to establish certain transformations for any non-developable surface that bears a family of asymptotic curves and is immersed in a space of $n$ dimensions $S_{n}(n>3)$. All surfaces mentioned hereafter will belong to $S_{n}$.

The ambient of the osculating planes at a point of a surface to all of the curves on the surface that go through the point is a space of not more than five dimensions. $\uparrow$ The class of all surfaces in $S_{n}$ for which the ambient at all points is a space of four dimensions is divided into two subclasses. One of these subclasses is composed of all surfaces in $S_{n}$ that bear each a conjugate net of curves, while the other is composed of all surfaces in $S_{n}$ that sustain each a family of asymptotic curves.

In the classical transformations for a surface bearing a conjugate net, the two congruences of lines tangent to the curves of the net have played basic rôles. We shall assign a similar rôle to the $\infty^{2}$ lines tangent to the asymptotic curves of a family. Although the congruence of these lines contains only a one parameter family of developable surfaces, it will be defined as a parabolic congruence.

To facilitate discussion, a terminology for certain geometric relations is introduced.

DeFinition 1. The asymptotic curves of a given family are said to be autoconjugate to the lines of a parabolic congruence if the curves of the family lie on the developable surfaces of the congruence, provided that the surface which sustains the given family is not the focal surface of the congruence.

Definition 2. A family of asymptotic curves on a surface and a parabolic congruence, such that there is just one line of the congruence lying in each tangent plane of the surface and not passing through the point of contact, are harmonic to each other in case the developable surfaces of the parabolic congruence correspond to the curves of the family.

\footnotetext{
* Presented to the Society, November 27, 1936; received by the editors March 16, 1937.

$\dagger$ Lane, Projective Differential Geometry of Curves and Surfaces, University of Chicago Press, 1932 , p. 124.
} 
Definition 3. If two families of asymptotic curves are autoconjugate to the same parabolic congruence, they are said to be in relation $F^{\prime}$.

The transformation $F^{\prime}$ of families of asymptotic lines is an analogue of the well known transformation $F$ of conjugate systems.*

DEFINITION 4. If the points of a surface are in a one-to-one correspondence with $\infty^{2}$ straight lines, and if corresponding points and lines are in united position, the surface is said to be transversal to the lines.

In $\$ 1$ is developed a transformation of a family of asymptotic curves which is analogous to the transformation of Levy for conjugate nets, $\dagger$ while in \$2 is exhibited a method for determining all of the parabolic congruences autoconjugate to a given family of asymptotic curves. There is developed in $\S 3$ a method for determining a family of asymptotic curves in relation $F^{\prime}$ with a given family of curves of the same kind. A study is made in $\$ 4$ of the relation of several $F^{\prime}$ transforms of a given family of asymptotic curves by means of the same parabolic congruence. The relation of two $F^{\prime}$ transforms of a given surface by means of two different parabolic congruences is considered in $\$ 5$, and a theorem of permutability for the transformation $F^{\prime}$ is established in $\S 6$. General transversal, surfaces of a parabolic congruence are examined in $\$ 7$, and it is proved that these surfaces too are transformable by some of the methods which we have applied to surfaces bearing families of asymptotic curves.

1. A transformation of a family of asymptotic curves. Consider a nondevelopable surface $S$ which sustains a family of asymptotic curves and is immersed in a projective space of $n$ dimensions $(n>3)$. A parametric vector equation of the surface may be written in terms of homogeneous coordinates as $y=y(u, v)$. We adopt the asymptotic curves as the $u$-curves, and any other family of curves on the surface as $v$-curves. The resulting coordinates $y$ of the generating point of the surface are known to satisfy a differential equation of the form

$$
y_{u u}=a y_{u}+b y_{v}+c y \quad(b \neq 0),
$$

called the point differential equation of the surface. The surface $S$ is any integral surface of equation (1.1).

To obtain a transformation of $S$, let $R$ be a solution of (1.1). The point determined by the coordinates

$$
x=R y_{u}-R_{u} y
$$

is on the line which is tangent at the point $y$ to the $u$-curve of $S$. We shall

* See Eisenhart, Transformations of Surfaces, Princeton University Press, 1923, p. 34.

† See Eisenhart, loc. cit., p. 19. 
show that the point $x$ generates a surface having a family of asymptotic curves as $u$-curves.

By computing derivatives of (1.2) and reducing them by means of (1.1), we obtain the relations

$$
\begin{aligned}
x & =R y_{u}-R_{u} y, \\
x_{u}-a x & =b\left(R y_{v}-R_{v} y\right), \\
x_{u u}-a x_{u}-b x_{v}-a_{u} x & =-2 b R_{v} y_{u}+\left(2 b R_{u}+b_{u} R\right) y_{v}-b_{u} R_{v} y .
\end{aligned}
$$

The determinant of the coefficients of $y, y_{u}$, and $y_{v}$ in the right members of (1.3) is equal to zero. Hence the left members satisfy a linear relation. This relation is equivalent to the differential equation

$$
x_{u u}=\left(a+\frac{b_{u}}{b}+\frac{2 R_{u}}{R}\right) x_{u}+b x_{v}+\left(a_{u}-\frac{2 a R_{u}}{R}-\frac{a b_{u}}{b}-\frac{2 b R_{v}}{R}\right) x,
$$

which indicates that the $u$-curves of the surface $S(x)$ belong to a family of asymptotic curves.

The first of equations (1.3) indicates that the surface $S(x)$ is transversal to the parabolic congruence of lines which are tangent to the $u$-curves of $S(y)$. Moreover, the family of asymptotic $u$-curves on $S(x)$ is autoconjugate to the parabolic congruence.

From the first and second equations of (1.3), we observe that the line which is tangent to the $u$-curve of $S(x)$ at the point $x$, lies in the plane which is tangent to $S(y)$ at the point $y$. Hence the parabolic congruence of lines tangent to the $u$-curves of $S(x)$ is harmonic to the family of asymptotic $u$-curves on $S(y)$.

The transformation (1.2) is an analogue of the transformation of Levy for conjugate nets. Repeated application of this transformation produces a sequence of surfaces which is a close analogue of a Levy sequence of conjugate nets.

We shall now prove that all families of asymptotic curves that are autoconjugate to the parabolic congruence of lines tangent to the $u$-curves of $S(y)$ are obtained by transformations of the same form as (1.2).

Let the generating point of a surface transversal to the tangent lines of the $u$-curves of $S(y)$ have coordinates $\xi$. By means of a transformation $y=\theta \eta$, let new coordinates $\eta$ for the generating point of $S(y)$ be chosen so that

$$
\xi=\eta_{u} \text {. }
$$

This change of coordinates transforms the differential equation (1.1) into an equation of the form 


$$
\eta_{u u}=\alpha \eta_{u}+\beta \eta_{v}+\gamma \eta
$$

in which certain of the coefficients are specialized by the particular choice of $\theta$.

In order that the surface $S(\xi)$ have a family of asymptotic $u$-curves, it is necessary and sufficient that $\xi$ satisfy an equation of the same form as (1.1). If, by means of equations (1.5) and (1.6) we express $\xi, \xi_{u}, \xi_{v}$, and $\xi_{u u}$ in terms of $\eta, \eta_{u}, \eta_{v}$, and $\eta_{u v}$, and set the determinant of the coefficients of the latter functions equal to zero, we find that

$$
\beta \gamma_{u}-\gamma \beta_{u}=0
$$

is the only restriction on the coefficients of (1.6) in order that $S(\xi)$ have the required family of asymptotic curves.

The general solution of (1.7) is $\gamma=\beta f(v)$, a special case of which is $\gamma=f(v)=0$. But in order for $\gamma$ to be zero, the above function $\theta$ must be a solution of (1.1). Then the coordinates $\xi$ can be written as

$$
\xi=\left(1 / \theta^{2}\right)\left(\theta y_{u}-\theta_{u} y\right) .
$$

If $f(v) \neq 0$, we introduce the value of $\gamma$, and verify that

$$
\xi_{u}-\alpha \xi=\beta\left(\eta_{v}+f \eta\right) .
$$

A second transformation $\eta=\mu(v) \zeta$ is introduced, where $\mu$ satisfies the condition $d \mu / d v=-\mu f$. As a result of this transformation, the above equation and (1.5) become

$$
\begin{aligned}
\xi_{u}-\alpha \xi & =\beta \mu \zeta_{v}, \\
\xi & =\mu \zeta_{u} .
\end{aligned}
$$

Integrability conditions on the left members of (1.8) show that $\zeta$ satisfies a differential equation of the same form as (1.6), but with $\gamma=0$.

The above transformations $y=\theta \eta$ and $\eta=\mu \zeta$ are equivalent to the single transformation $y=R \zeta$. Since this transformation changes (1.1) into a new equation in $\zeta$, of the same form as (1.6), but with $\gamma=0$, the function $R$ is a solution of (1.1).

The second of equations (1.8) can be written in the form $\xi$ $=\left(\mu / R^{2}\right)\left(R y_{u}-R_{u} y\right)$. This establishes

THEOREM 1. Let $S(y)$ be a surface bearing a family of asymptotic u-curves, and for which the point differential equation is (1.1). Let $S(x)$ be any surface which sustains a family of asymptotic curves autoconjugate to the parabolic congruence of lines tangent to the u-curves of $S(y)$. Then the transformation which sends $S(y)$ into $S(x)$ may be represented by a relation of the form $x=R y_{u}-R_{u} y$, in which $R$ is a solution of (1.1). 
We make use of a second solution $R^{\prime}$ of equation (1.1) to construct the transformation of $S(x)$ which is represented by the equation

$$
x^{\prime}=R^{\prime} y_{u}-R_{u}^{\prime} y .
$$

From equations (1.1) and (1.9) is derived the relation

$$
x_{u}^{\prime}-a x^{\prime}=b\left(R^{\prime} y_{v}-R_{v}^{\prime} y\right),
$$

which is similar to the second of (1.3). Equations (1.9) and (1.10) together with (1.3) indicate that the line tangent at the point $x^{\prime}$ to the $u$-curve of $S\left(x^{\prime}\right)$, and the corresponding line tangent to the $u$-curve of $S(x)$, lie in the plane which is tangent at the point $y$ to $S(y)$. Since these lines lie in a plane, they intersect in a point having coordinates $x^{\prime \prime}$. We shall prove that the point $x^{\prime \prime}$ generates a surface which has a family of asymptotic lines as parametric $u$-curves, and that this family of curves is autoconjugate to each of the parabolic congruences formed by the lines tangent to the $u$-curves of $S(x)$ and $S\left(x^{\prime}\right)$ respectively.

Since $R^{\prime}$ is a solution of (1.1), the function

$$
\theta=R R_{u}^{\prime}-R^{\prime} R_{u} .
$$

is a solution of the point differential equation of $S(x)$. This fact follows from replacing $x$ by $R^{\prime}$ in (1.2). For a similar reason, the function

$$
\theta^{\prime}=R^{\prime} R_{u}-R R_{u}^{\prime}
$$

is a solution of the point differential equation of $S\left(x^{\prime}\right)$. We define $\psi=R R_{v}^{\prime}$ $-R_{v} R^{\prime}$. Then by (1.11) and (1.1),

$$
\begin{aligned}
\theta_{u} & =a \theta+b \psi, \\
\theta_{u} & =a \theta^{\prime}-b \psi .
\end{aligned}
$$

To determine the coordinates of the point of intersection of the two corresponding tangent lines to the $u$-curves of $S(x)$ and $S\left(x^{\prime}\right)$, we list the following relations

$$
\begin{aligned}
x & =R y_{u}-R_{u} y, \\
x_{u} & =R\left(a y_{u}+b y_{v}\right)-\left(a R_{u}+b R_{v}\right) y, \\
x^{\prime} & =R^{\prime} y_{u}-R_{u}^{\prime} y, \\
x_{u}^{\prime} & =R^{\prime}\left(a y_{u}+b y_{v}\right)-a\left(R_{u}^{\prime}+b R_{v}^{\prime}\right) y .
\end{aligned}
$$

By eliminating $y, y_{u}$, and $y_{v}$ from (1.14), we obtain the relation

$$
R^{\prime}\left[\theta x_{u}-(a \theta+b \psi) x\right]=-R\left[\theta^{\prime} x_{u}^{\prime}-\left(a \theta^{\prime}-b \psi\right) x^{\prime}\right]
$$


After using equations (1.13) to reduce the coefficients of $x$ and $x^{\prime}$ in the above equation, we have the coordinates

$$
x^{\prime \prime}=R^{\prime}\left(\theta x_{u}-\theta_{u} x\right)=-R\left(\theta^{\prime} x_{u}^{\prime}-\theta_{u}^{\prime} x^{\prime}\right) .
$$

Since $\theta$ and $\theta^{\prime}$ are solutions of the point differential equations of $S(x)$ and $S\left(x^{\prime}\right)$ respectively, the surface $S\left(x^{\prime \prime}\right)$ is by (1.2) a transform of $S(x)$ and $S\left(x^{\prime}\right)$ alike. By virtue of these transformations, $S\left(x^{\prime \prime}\right)$ has a family of asymptotic curves as $u$-curves, and this family is autoconjugate to each of the two parabolic congruences formed by lines tangent to the $u$-curves of the surfaces $S(x)$ and $S\left(x^{\prime}\right)$ respectively.

Since, by the remark following equation (1.10), the line tangent at the point $x^{\prime \prime}$ to the $u$-curve of $S\left(x^{\prime \prime}\right)$ lies in the plane tangent at the point $x$ to $S(x)$, and lies also in the plane tangent at the point $x^{\prime}$ to $S\left(x^{\prime}\right)$, it follows that the families of asymptotic curves of both $S(x)$ and $S\left(x^{\prime}\right)$ are harmonic to the parabolic congruence formed by the totality of these tangent lines. From these results, we state

THEOREM 2. If two families of asymptotic curves are autoconjugate to the same parabolic congruence, they are both harmonic to a second parabolic congruence.

2. Parabolic congruences autoconjugate to a family of asymptotic curves. Let $S(x)$ be a surface having a family of asymptotic $u$-curves. Denote by $S(y)$ the focal surface of a parabolic congruence that is autoconjugate to the family of asymptotic curves on $S(x)$.

The coordinates $x$ satisfy a differential equation

$$
x_{u u}=a x_{u}+b x_{v}+c x,
$$

and the coordinates $y$ satisfy an equation

$$
y_{u u}=A y_{u}+B y_{v}+C y \text {. }
$$

Since the point $x$ is on the line tangent at point $y$ to the $u$-curve of $S(y)$, the coordinates $x$ and $y$ satisfy a relation

$$
y_{u}+\lambda y=\mu x \text {. }
$$

To obtain the consequences of equations (2.1), (2.2), and (2.3), we take the derivative with respect to $u$ of (2.3), and reduce the result by (2.2) and (2.3). The relation reduces to the equation

$$
B y_{v}+\left(C+\lambda_{u}-\lambda^{2}-A \lambda\right) y=\mu x_{u}+\left(\mu_{u}-\mu A-\mu \lambda\right) x,
$$

which can be written in an abbreviated form with (2.3) to give the system 


$$
\begin{aligned}
& y_{u}+\alpha y=\beta x, \\
& y_{v}+\rho y=\sigma x_{u}+\tau x .
\end{aligned}
$$

Equations (2.4) imply relations of the form (2.2) and (2.3). To demonstrate this fact, we compute the derivative with respect to $u$ of the first equation of (2.4) and eliminate $x$.

Henceforth we shall investigate the consequences of equations (2.4) in view of (2.1). On differentiating (2.4) we obtain the system

$$
\begin{aligned}
y_{u}+\alpha y & =\beta x, \\
y_{u v}+\alpha y_{v}+\alpha_{v} y & =\beta x_{v}+\beta_{v} x, \\
y_{v}+\rho y & =\sigma x_{u}+\tau x, \\
y_{u v}+\rho y_{u}+\rho_{u} y & =\left(\sigma_{u}+a \sigma+\tau\right) x_{u}+\sigma b x_{v}+\left(\tau_{u}+\sigma c\right) x .
\end{aligned}
$$

The right members of these equations are linearly dependent. Unless the determinant of the coefficients of the left members vanishes there exists a linear relation in the functions $y_{u v}, y_{u}, y_{v}$, and $y$. Such a relation together with (2.2) would restrict the surface $S(y)$ to be developable. We consider the case for which $S(y)$ is not developable, and for which the determinant vanishes. The expanded form of the determinant set equal to zero is

$$
\rho_{u}-\alpha_{v}=0 .
$$

This equation makes it possible, by means of a transformation $y=\phi \bar{y}$, to reduce equations (2.4) to the form

$$
\begin{aligned}
& y_{u}=g x, \\
& y_{v}=R x_{u}+S x .
\end{aligned}
$$

On applying integrability conditions to the left members of (2.7), we obtain the relation

$$
x_{u}\left(R a+R_{u}+S\right)+x_{v}(R b-g)+x\left(S_{u}+R c-g_{v}\right)=0 .
$$

The left member of this equation must vanish identically in $x$ and its derivatives. The coefficients set equal to zero give

$$
\begin{aligned}
S & =-\left(R_{u}+R a\right), \\
g & =R b, \\
S_{u} & =g_{v}-R c .
\end{aligned}
$$

By using the first and second equations of (2.8) to eliminate $g$ and $S$ from the third, we show that $R$ is a solution of the equation

$$
R_{u u}=-a R_{u}-b R_{v}+\left(c-a_{u}-b_{v}\right) R .
$$

These results are summarized in the following theorem. 
THEOREM 3. Let there be given an integral surface $S(x)$ of the point differential equation (2.1), and let $R$ be a solution of (2.9), with $g$ and $S$ determined by the first two of equations (2.8). Then the coordinates y obtained by quadratures from (2.7) determine the generating point of the focal surface of a parabolic congruence that is autoconjugate to the family of asymptotic u-curves on $S(x)$.

3. Families of asymptotic curves in relation $F^{\prime}$. Two families of asymptotic curves which are autoconjugate to the same parabolic congruence are, by Definition 3, in relation $F^{\prime}$.

Let $S(x)$ and $S\left(x^{\prime}\right)$ be two surfaces bearing each a family of asymptotic curves, and so related that the two families are in relation $F^{\prime}$. Let $S(y)$ be the focal surface of the parabolic congruence to which they are autoconjugate, the point differential equation of this surface being

$$
y_{u u}=\alpha y_{u}+\beta y_{v}+\gamma y \text {. }
$$

By reason of Theorem 1, the coordinates $x$ and $x^{\prime}$ can be chosen so as to satisfy the following relations

$$
\begin{aligned}
x & =R y_{u}-R_{u} y, \\
x_{u}-\alpha x & =\beta\left(R y_{v}-R_{v} y\right), \\
x^{\prime} & =R^{\prime} y_{u}-R_{u}^{\prime} y, \\
x_{u}^{\prime}-\alpha x^{\prime} & =\beta\left(R^{\prime} y_{v}-R_{v}^{\prime} y\right),
\end{aligned}
$$

in which $R$ and $R^{\prime}$ are solutions of (3.1) for which $R R_{u}^{\prime}-R^{\prime} R_{u} \neq 0$.

As a result of eliminating $y$ and its derivatives from (3.2), we obtain the following relations:

$$
\begin{aligned}
& x_{u}-A x=M x_{u}^{\prime}+N x^{\prime}, \\
& x_{v}-B x=P x_{u}^{\prime}+M x_{v}^{\prime}+Q x^{\prime} .
\end{aligned}
$$

The coordinates $x$ satisfy an equation of the form

$$
x_{u u}=a x_{u}+b x_{v}+c x,
$$

and the coordinates $x^{\prime}$ satisfy a similar one. By computing the derivative with respect to $v$ of the first of (3.3), and the derivative with respect to $u$ of the second, and reducing the resulting system by (3.4), it can be shown that unless the determinant $A_{v}-B_{u}$ is equal to zero, the point $x$ lies in the plane tangent to $S\left(x^{\prime}\right)$ at the point $x^{\prime}$.

With the condition $A_{v}-B_{u}=0$, equations (3.3) are transformed by the substitution $x=\theta \bar{x}$ into the form 


$$
\begin{aligned}
& \bar{x}_{u}=M x_{u}^{\prime}+N x^{\prime}, \\
& \bar{x}_{v}=P x_{u}^{\prime}+M x_{v}^{\prime}+Q x^{\prime} .
\end{aligned}
$$

On taking suitable linear combinations of (3.5), we obtain the relations

$$
\begin{aligned}
x_{u}^{\prime}-A^{\prime} x^{\prime} & =\bar{M} \bar{x}_{u}, \\
x_{v}^{\prime}-B^{\prime} x^{\prime} & =\bar{P} \bar{x}_{u}+\bar{M} \bar{x}_{v} .
\end{aligned}
$$

An argument similar to the above suffices to show that these equations can be transformed, by a substitution $x^{\prime}=\theta^{\prime} \bar{x}^{\prime}$, so that $\bar{A}^{\prime}=\bar{B}^{\prime}=0$. We therefore write

$$
\begin{aligned}
& \bar{x}_{u}^{\prime}=m \bar{x}_{u}, \\
& \bar{x}_{v}^{\prime}=p \bar{x}_{u}+m \bar{x}_{v} .
\end{aligned}
$$

Integrability conditions applied to the left members of (3.6) lead to a differential equation in $\bar{x}$ of the form

$$
\bar{x}_{u u}=\bar{a} \bar{x}_{u}+b \bar{x}_{v},
$$

where

$$
\begin{aligned}
& m_{u}=-p \bar{b}, \\
& m_{v}=p_{u}+\bar{a} p .
\end{aligned}
$$

We observe that in order to reduce (3.4) to the form (3.7) iby a transformation $x=\theta \bar{x}$, it is necessary and sufficient that $\theta$ be a solution of (3.4). Coefficients of (3.7) are $\bar{a}=a-2 \theta_{u} / \theta, b=b$.

By eliminating $m$ from (3.8) it is found that $p$ satisfies the equation

$$
p_{u u}+\bar{a} p_{u}+\bar{b} p_{v}+\left(\bar{a}_{u}+\bar{b}_{v}\right) p=0 .
$$

We have in conclusion

THEOREM 4. Let $S(x)$ be an integral surface of (3.4), and let $\theta, p$, and $m$ be solutions of (3.4), (3.9), and (3.8) respectively. Then the coordinates $x^{\prime}$ obtained by quadratures from the equations

$$
\begin{aligned}
& x_{u}^{\prime}=m\left(\frac{x}{\theta}\right)_{u}, \\
& x_{v}^{\prime}=p\left(\frac{x}{\theta}\right)_{u}+m\left(\frac{x}{\theta}\right)_{v},
\end{aligned}
$$

determine the generating point of a surface $S\left(x^{\prime}\right)$ having a family of asymptotic $u$-curves in relation $F^{\prime}$ with the family of asymptotic u-curves on $S(x)$.

One readily verifies that the point having coordinates $\eta=x^{\prime}-m(x / \theta)$ is 
the generating point of the focal surface of the parabolic congruence autoconjugate to the asymptotic curves on $S(x)$ and $S\left(x^{\prime}\right)$.

4. Transformations $F^{\prime}$ with the same parabolic congruence. Let $S(x)$ and $S\left(x^{\prime}\right)$ be two surfaces with families of asymptotic $u$-curves in relation $F^{\prime}$, as represented by the equations

$$
\begin{aligned}
& x_{u}^{\prime}=m x_{u}, \\
& x_{v}^{\prime}=p x_{u}+m x_{v} .
\end{aligned}
$$

The coordinates $x$ satisfy a differential equation

$$
x_{u u}=a x_{u}+b x_{v} .
$$

In order to determine on a third surface a family of asymptotic curves that is autoconjugate to the parabolic congruence of lines which connect corresponding points of $S(x)$ and $S\left(x^{\prime}\right)$, we make the change of coordinates

$$
x^{\prime}=\theta^{\prime} x_{1}^{\prime}
$$

and define the coordinates of a point on the line $x x^{\prime}$ as

$$
x_{1}=x-\theta x_{1}^{\prime} \text {. }
$$

In terms of the old coordinates

$$
x_{1}=x-\frac{\theta}{\theta^{\prime}} x^{\prime} .
$$

The functions $\theta$ and $\theta^{\prime}$ will be determined so that $x_{1}$ and $x_{1}^{\prime}$ satisfy a system of equations similar to (4.1).

By differentiating (4.3) and (4.4), and applying (4.1), we establish the equations

$$
\begin{aligned}
& x_{1 u}=\frac{1}{m}\left[x_{1 u}^{\prime}\left(\theta^{\prime}-m \theta\right)+x_{1}^{\prime}\left(\theta_{u}^{\prime}-m \theta_{u}\right)\right], \\
& x_{1 v}=\frac{1}{m}\left[x_{1 v}^{\prime}\left(\theta^{\prime}-\theta\right) m-x_{1 u}^{\prime} \frac{p \theta^{\prime}}{m}+x_{1}^{\prime}\left(\theta_{v}^{\prime}-m \theta_{v}-\frac{p}{m} \theta_{u}^{\prime}\right)\right] .
\end{aligned}
$$

If these equations are to take the form of (4.1), the coefficients of $x_{1}^{\prime}$ must vanish. This gives the conditions

$$
\begin{aligned}
& \theta_{u}^{\prime}=m \theta_{u}, \\
& \theta_{v}^{\prime}=p \theta_{u}+m \theta_{v} .
\end{aligned}
$$

From the integrability conditions on (4.6), $\theta$ is a solution of (4.2), and $\theta^{\prime}$ is obtained by quadratures on (4.6). The function $\theta^{\prime}$ is a solution of the point 
differential equation of $S\left(x^{\prime}\right)$. It can also be verified that

$$
\begin{aligned}
& \frac{\partial}{\partial u}\left(\frac{x_{1} \theta^{\prime}}{\theta}\right)=\left(\theta^{\prime}-m \theta\right) \frac{\partial}{\partial u}\left(\frac{x}{\theta}\right), \\
& \frac{\partial}{\partial v}\left(\frac{x_{1} \theta^{\prime}}{\theta}\right)=\left(\theta^{\prime}-m \theta\right) \frac{\partial}{\partial v}\left(\frac{x}{\theta}\right)-p \theta \frac{\partial}{\partial u}\left(\frac{x}{\theta}\right) .
\end{aligned}
$$

The above deductions justify the following theorem:

THEOREM 5. Let there be given two surfaces $S(x)$ and $S\left(x^{\prime}\right)$ sustaining families of asymptotic curves in relation $F^{\prime}$, and let the coordinates $x$ and $x^{\prime}$ be chosen so that equations (4.1) and (4.2) hold. Then in terms of $\theta, a$ solution of (4.2), and $\theta^{\prime}$, a corresponding solution of (4.6), the coordinates $x_{1}$ defined by (4.4) determine the generating point of a surface $S\left(x_{1}\right)$ which has a family of asymptotic $u$-curves in relation $F^{\prime}$ with the corresponding families on $S(x)$ and $S\left(x^{\prime}\right)$.

As an example of the above transformation, we shall establish the following theorem:

THEOREM 6. If a surface $S(x)$ bearing a family of asymptotic curves lies on a hyperquadric, any parabolic congruence autoconjugate to the family meets the hyperquadric again in a surface bearing a family of asymptotic curves in relation $F^{\prime}$ with the first family.

Let the equation of the hyperquadric be written as

$$
\sum a_{i k} x^{i} x^{k}=0 \text {. }
$$

Let the chosen parabolic congruence be autoconjugate to the family of asymptotic curves on $S\left(x^{\prime}\right)$, where the coordinates $x^{\prime}$ of the generating point of $S\left(x^{\prime}\right)$ are defined by (4.1). From differentiating (4.8) and reducing the results by (4.2), we obtain the relations

$$
\sum a_{i k}\left(x_{u}^{i} x^{k}+x^{i} x_{u}^{k}\right)=\sum a_{i k}\left(x_{v}^{i} x^{k}+x^{i} x_{v}^{k}\right)=\sum a_{i k} x_{u}^{i} x_{u}^{k}=0 .
$$

By means of these relations, it can be shown that the function

$$
\theta=\sum a_{i k}\left(x^{i} x^{\prime k}+x^{\prime i} x^{k}\right)
$$

is a solution of the point equation (4.2) of $S(x)$, and that the function

$$
\theta^{\prime}=\sum a_{i k} x^{\prime i} x^{\prime k}
$$

is a corresponding solution of (4.6).

If $S\left(x_{1}\right)$ is determined by the transformation (4.4), using $\theta$ and $\theta^{\prime}$ from (4.9) and (4.10), it is easy to show that $x_{1}$ satisfies the equation (4.8) of the hyperquadric. The surfaces $S\left(x_{1}\right)$ and $S(x)$ are in relation $F^{\prime}$ by transformation (4.4). 
As a second example of the transformation, we consider

THEOREM 7. If a transversal surface of a parabolic congruence lies in a hyperplane, it has on it a family of asymptotic curves that is autoconjugate to the parabolic congruence.

Let the given surface be transversal to the parabolic congruence of lines that join corresponding points of $S(x)$ and $S\left(x^{\prime}\right)$, and define the coordinates of its generating point as

$$
\xi=x-\lambda x^{\prime} .
$$

The equation of the hyperplane may be taken as $\xi^{i}=0$. If $\lambda$ is determined so that $\xi^{i}=0$, its value is $\lambda=x^{i} / x^{\prime i}$. As a consequence

$$
\xi=x-\frac{x^{i}}{x^{\prime i}} x^{\prime}
$$

The functions $x^{i}$ and $x^{\prime i}$ have the properties of $\theta$ and $\theta^{\prime}$ which are required by the $F^{\prime}$ transformation (4.4). Hence $S(\xi)$ is an $F^{\prime}$ transform of each of $S(x)$ and $S\left(x^{\prime}\right)$.

For the $F^{\prime}$ transformation (4.4), which sends $S(x)$ into $S\left(x_{1}\right)$ by means of the auxiliary surface $S\left(x^{\prime}\right) \equiv S\left(x_{1}^{\prime}\right)$, we wish to determine an inverse. That is, we wish to determine a pair of functions $\theta^{-1}$ and $\left(\theta^{\prime}\right)^{-1}$ such that

$$
x=x_{1}-\frac{\theta^{-1}}{\left(\theta^{\prime}\right)^{-1}} x_{1}^{\prime},
$$

where $\theta^{-1}$ is a solution of the point differential equation of $S\left(x_{1}\right)$, and $\left(\theta^{\prime}\right)^{-1}$ is related to $\theta^{-1}$ by equations similar to (4.6) which give the relations between $\theta^{\prime}$ and $\theta$. Analogues of (4.6) are obtained by solving (4.5), in view of (4.6), for $x_{1 u}^{\prime}$ and $x_{10}^{\prime}$, then replacing $x_{1}^{\prime}$ by $\left(\theta^{\prime}\right)^{-1}$ and $x_{1}$ by $\theta^{-1}$. The equations are

$$
\begin{aligned}
& \left(\theta^{\prime}\right)_{u^{-1}}=\frac{m}{\theta^{\prime}-m \theta} \theta_{u}^{-1}, \\
& \left(\theta^{\prime}\right)_{v}^{-1}=\frac{p \theta^{\prime}}{\left(\theta^{\prime}-m \theta\right)^{2}} \theta_{u}^{-1}+\frac{m}{\theta^{\prime}-m \theta} \theta_{v}^{-1} .
\end{aligned}
$$

Equations (4.13), as well as the point differential equations of $S\left(x_{1}\right)$ and $S\left(x_{1}^{\prime}\right)$ obtainable from them by integrability conditions, are satisfied by the functions

$$
\theta^{-1}=-\frac{\theta}{\theta^{\prime}}, \quad\left(\theta^{\prime}\right)^{-1}=\frac{1}{\theta^{\prime}}
$$


These functions also give a result consistent with (4.4) when they are substituted into (4.12). It can be verified that

$$
\begin{aligned}
& \frac{\partial}{\partial u}\left(\frac{1}{\theta^{-1}}\right)=\left(m \theta-\theta^{\prime}\right) \frac{\partial}{\partial u}\left(\frac{1}{\theta}\right), \\
& \frac{\partial}{\partial v}\left(\frac{1}{\theta^{-1}}\right)=\left(m \theta-\theta^{\prime}\right) \frac{\partial}{\partial v}\left(\frac{1}{\theta}\right)+p \theta \frac{\partial}{\partial u}\left(\frac{1}{\theta}\right) .
\end{aligned}
$$

Theorem 8. If $S(x)$ is transformed into $S\left(x_{1}\right)$ by means of $\theta, \theta^{\prime}$, and the auxiliary surface $S\left(x^{\prime}\right)$, as expressed in (4.4), then $S\left(x_{1}\right)$ is transformed into $S(x)$ by means of the functions $\theta^{-1},\left(\theta^{\prime}\right)^{-1}$, and $x_{1}^{\prime}$, where $\theta^{-1},\left(\theta^{\prime}\right)^{-1}$, and $x_{1}^{\prime}$ are defined by (4.14) and (4.3), and the transformation is expressed by (4.12).

If by means of a second solution $\theta_{2}$ of the point equation (4.2), $S(x)$ is transformed into a second surface $S\left(x_{2}\right)$ by the relation

$$
x_{2}=x-\frac{\theta_{2}}{\theta_{2}^{\prime}} x^{\prime},
$$

the surfaces $S\left(x_{1}\right)$ and $S\left(x_{2}\right)$ are in relation $F^{\prime}$. It is useful to know by what analytical relation $S\left(x_{1}\right)$ is transformed into $S\left(x_{2}\right)$. By subtracting equation (4.4) from (4.16) we obtain

$$
x_{2}=x_{1}-\frac{\theta_{3} x^{\prime}}{\theta_{3}^{\prime} \theta^{\prime}},
$$

where the function

$$
\theta_{3}=\theta_{2}-\frac{\theta}{\theta^{\prime}} \theta_{2}^{\prime}
$$

is by (4.4) a solution of the point equation of $S\left(x_{1}\right)$, and the function

$$
\theta_{3}^{\prime}=\frac{\theta_{2}^{\prime}}{\theta^{\prime}}
$$

is a solution of the point equation of $S\left(x^{\prime} / \theta^{\prime}\right)$.

5. Transformations $F^{\prime}$ by two congruences. Let $S\left(x^{\prime}\right)$ and $S\left(x^{\prime \prime}\right)$ be two $F^{\prime}$ transforms of $S(x)$, where the differential equations of the transformations are

$$
\begin{aligned}
x_{u}^{\prime} & =m_{1} x_{u}, \quad x_{v}^{\prime}=p_{1} x_{u}+m_{1} x_{v} ; \\
x_{u}^{\prime \prime} & =m_{2} x_{u}, \quad x_{v}^{\prime \prime}=p_{2} x_{u}+m_{2} x_{v},
\end{aligned}
$$

in which the coordinates $x$ satisfy (4.2). By means of $S\left(x^{\prime}\right), S\left(x^{\prime \prime}\right)$, and a 
solution $\theta_{1}$ of (4.2) we obtain two more $F^{\prime}$ transforms of $S(x)$, which are represented by the equations

$$
x_{1,1}=x-\frac{\theta_{1}}{\theta_{1}^{\prime}} x^{\prime}, \quad x_{1,2}=x-\frac{\theta_{1}}{\theta_{1}^{\prime \prime}} x^{\prime \prime},
$$

in which $\theta_{1}^{\prime}$ and $\theta_{1}^{\prime \prime}$ are obtained from (5.1) by replacing $x$ by $\theta$ throughout.

There is an $F^{\prime}$ transform of $S\left(\dot{x}^{\prime \prime}\right)$ by means of $\theta_{1}^{\prime \prime}$ and $S\left(x^{\prime}\right)$. The coordinates of its generating point are determined by the equation

$$
x_{1,1}^{\prime \prime \prime}=x^{\prime \prime}-\frac{\theta_{1}^{\prime \prime}}{\theta_{1}^{\prime}} x^{\prime}
$$

It is easy to show that the point $x_{1,1}^{\prime \prime \prime}$ is the intersection of the lines $x^{\prime} x^{\prime \prime}$ and $x_{1,1} x_{1,2}$.

By differentiating (5.3) and the first of (5.2), we can establish, at the end of some labor, the following equations:

$$
\begin{aligned}
\frac{\partial}{\partial u}\left(x_{1,1}^{\prime \prime \prime}\right)= & \frac{m_{2} \theta_{1}^{\prime}-m_{1} \theta_{1}^{\prime \prime}}{\theta_{1}^{\prime}-m_{1} \theta_{1}} \frac{\partial}{\partial u}\left(x_{1,1}\right), \\
\frac{\partial}{\partial v}\left(x_{1,1}^{\prime \prime \prime}\right)= & {\left[\frac{m_{2} \theta_{1}^{\prime}-m_{1} \theta_{1}^{\prime \prime}}{\left(\theta_{1}^{\prime}-m_{1} \theta_{1}\right)^{2}} p_{1} \theta_{1}+\frac{p_{2} \theta_{1}^{\prime}-p_{1} \theta_{1}^{\prime \prime}}{\theta_{1}^{\prime}-m_{1} \theta_{1}}\right] \frac{\partial}{\partial u}\left(x_{1,1}\right) } \\
& +\frac{m_{2} \theta_{1}^{\prime}-m_{1} \theta_{1}^{\prime \prime}}{\theta_{1}^{\prime}-m_{1} \theta_{1}} \frac{\partial}{\partial v}\left(x_{1,1}\right) .
\end{aligned}
$$

These equations show that $S\left(x_{1,1}^{\prime \prime}\right)$ and $S\left(x_{1,1}\right)$ are in relation $F^{\prime}$. It follows that $S\left(x_{1,1}^{\prime \prime \prime}\right)$ and $S\left(x_{1,2}\right)$ are in relation $F^{\prime}$.

An inverse of the $F^{\prime}$ transformation (4.4) is given by (4.12) in view of (4.14). On adapting these equations to the first of (5.2) we determine $-\theta_{1} / \theta_{1}^{\prime}$ as a solution of the point differential equation of $S\left(x_{1,1}\right)$. If we set $x_{1,1}=-\theta_{1} / \theta_{1}^{\prime}$ in (5.4), there is obtainable by quadrature a corresponding solution of the point equation of $S\left(x_{1,1}^{\prime \prime \prime}\right)$. We verify that $x_{1,1}^{\prime \prime \prime}=-\theta_{1}^{\prime \prime} / \theta_{1}^{\prime}$ is such a solution. Using these two solutions, we construct the transformation

$$
x_{1,2}^{\prime}=x_{1,1}-\frac{\theta_{1}}{\theta_{1}^{\prime \prime}} x_{1,1}^{\prime \prime \prime} \text {. }
$$

It can be shown that $x_{1,2}^{\prime} \equiv x_{1,2}$ by means of equations (5.2) and (5.3). These results are summarized in

THEOREM 9. If a family of asymptotic curves on $S(x)$ is transformed into two other families of asymptotic curves on $S\left(x_{1,1}\right)$ and $S\left(x_{1,2}\right)$ respectively by means of the same function $\theta_{1}$, the latter two families are in relation $F^{\prime}$; moreover, 
any two of the three families $S(x), S\left(x_{1,1}\right), S\left(x_{1,2}\right)$ are transforms of the third by means of the same solution of the point differential equation of the third.

The three families form a close analogy to a triad of conjuage nets.*

6. A theorem of permutability of transformation $F^{\prime}$. From (5.2) it can be seen that a solution of the point differential equation of $S\left(x_{1,1}\right)$ is given by

$$
\theta_{12}=\theta_{2}-\frac{\theta_{1}}{\theta_{1}^{\prime}} \theta_{2}^{\prime}
$$

where $\theta_{2}$ is a solution of (4.2), and $\theta_{2}^{\prime}$ is a corresponding solution of the first pair of (5.1). To get a solution of the point equation of $S\left(x_{1,1}^{\prime \prime \prime}\right)$, let $\theta_{2}^{\prime \prime}$ be a solution of the second pair of (5.1) corresponding to $x=\theta_{2}$; then from (5.3) we have the desired solution

$$
\theta_{12}^{\prime \prime \prime}=\theta_{2}^{\prime \prime}-\frac{\theta_{1}^{\prime \prime}}{\theta_{1}^{\prime}} \theta_{2}^{\prime}
$$

By means of the above solutions $\theta_{12}$ and $\theta_{12}^{\prime \prime \prime}$, we construct the following transformation of $S\left(x_{1,1}\right)$ :

$$
x_{(18)}=x_{1,1}-\frac{\theta_{2} \theta_{1}^{\prime}-\theta_{1} \theta_{2}^{\prime}}{\theta_{2}^{\prime \prime} \theta_{1}^{\prime}-\theta_{1}^{\prime \prime} \theta_{2}^{\prime}} x_{1,1}^{\prime \prime \prime} .
$$

Using the solution $\theta_{2}$, and equations similar to (5.2) we have two more transformations of $S(x)$ as follows:

$$
x_{2,1}=x-\frac{\theta_{2}}{\theta_{2}^{\prime}} x^{\prime}, \quad x_{2,2}=x-\frac{\theta_{2}}{\theta_{2}^{\prime \prime}} x^{\prime \prime} .
$$

Corresponding points of $S(x), S\left(x_{1,2}\right)$, and $S\left(x_{2,2}\right)$ are on a straight line. They are in relation $F^{\prime}$ by pairs. To determine the analytic relation by which $S\left(x_{1,2}\right)$ is transformed into $S\left(x_{2,2}\right)$ we compare equations (5.2) and (6.4) to (4.4) and (4.16), and draw conclusions corresponding to (4.17), (4.18), and (4.19). The results show that

$$
\theta_{2}-\frac{\theta_{1}}{\theta_{1}^{\prime \prime}} \theta_{2}^{\prime \prime}
$$

is the required solution of the point equation of $S\left(x_{1,2}\right)$, and that $\theta_{2}^{\prime \prime} / \theta_{1}^{\prime \prime}$ is a solution of the point equation of $S\left(x^{\prime \prime} / \theta_{1}^{\prime \prime}\right)$. That is, $S\left(x_{2,2}\right)$ is a transform of $S\left(x_{1,2}\right)$ by means of the solution (6.5).

From equations (5.5), placing $x_{1,2}^{\prime}=x_{1,2}$, and (6.3), it is seen that $x_{(12)}$ is a transform of $S\left(x_{1,2}\right)$ by means of the function

* Eisenhart, loc. cit., p. 44. 


$$
\theta_{12}-\frac{\theta_{1}}{\theta_{1}^{\prime \prime}} \theta_{12}^{\prime \prime \prime}
$$

which is a solution of the point equation of $S\left(x_{1,2}\right)$. This expression is reducible to (6.5) by (6.1) and (6.2). Hence we have shown that $S\left(x_{2,2}\right)$ and $S\left(x_{(12)}\right)$ are transforms of $S\left(x_{1,2}\right)$ by means of the same solution of its point differential equation. It follows that $S\left(x_{(12)}\right)$ and $S\left(x_{2,2}\right)$ are in relation $F^{\prime}$. We shall now investigate $S\left(x_{(12)}\right)$ through its relation to $S\left(x_{2,2}\right)$ and $S\left(x_{2,1}\right)$.

The point where the line $x^{\prime} x^{\prime \prime}$ intersects the line $x_{2,2} x_{2,1}$ has coordinates

$$
x_{2,2}^{\prime \prime \prime \prime}=x^{\prime}-\frac{\theta_{2}^{\prime}}{\theta_{2}^{\prime \prime}} x^{\prime \prime},
$$

which are obtained by subtracting equations (6.4). From equations (6.4) we obtain as a solution of the point differential equation of $S\left(x_{2,2}\right)$,

$$
\theta_{21}=\theta_{1}-\frac{\theta_{2}}{\theta_{2}^{\prime \prime}} \theta_{1}^{\prime} \text {. }
$$

A corresponding solution $\theta_{21}^{\prime \prime \prime}$ is obtained from (6.7) as

$$
\theta_{21}^{\prime \prime \prime}=\theta_{1}^{\prime}-\frac{\theta_{2}^{\prime}}{\theta_{2}^{\prime \prime}} \theta_{1}^{\prime \prime} .
$$

From these two solutions, we construct the transformation

$$
x_{(21)}=x_{2,2}-\frac{\theta_{1} \theta_{2}^{\prime \prime}-\theta_{2} \theta_{1}^{\prime \prime}}{\theta_{1}^{\prime} \theta_{2}^{\prime \prime}-\theta_{2}^{\prime} \theta_{1}^{\prime \prime}} x_{2,2}^{\prime \prime \prime} .
$$

Using the foregoing equations, it is easy to show that

$$
x_{(12)}-x=x_{(21)}-x=\frac{\left(\theta_{1}^{\prime \prime} \theta_{2}-\theta_{2}^{\prime \prime} \theta_{1}\right) x^{\prime}+\left(\theta_{2}^{\prime} \theta_{1}-\theta_{1}^{\prime} \theta_{2}\right) x^{\prime \prime}}{\theta_{1}^{\prime} \theta_{2}^{\prime \prime}-\theta_{1}^{\prime \prime} \theta_{2}^{\prime}} .
$$

In order to estimate the prevalence of the transformations $F^{\prime}$ that exist for given families of asymptotic curves, we count the constants in the foregoing quadratures. From the manner in which $\theta_{1}^{\prime}, \theta_{1}^{\prime \prime}, \theta_{2}^{\prime}, \theta_{2}^{\prime \prime}$ were obtained, each contains an arbitrary constant. If $S\left(x_{1,1}\right)$ and $S\left(x_{2,2}\right)$ are chosen transforms of $S(x)$, the constants in $\theta_{1}^{\prime}$ and $\theta_{2}^{\prime \prime}$ are determined by the choice. The constants in $\theta_{2}^{\prime}$ and $\theta_{2}^{\prime \prime}$ are left arbitrary. From these facts, we state for transformations $F^{\prime}$ a theorem of permutability.

THEOREM 10. If $S\left(x_{1,1}\right)$ and $S\left(x_{2,2}\right)$ are two $F^{\prime}$ transforms of $S(x)$ by means of functions $\theta_{1}$ and $\theta_{2}$, and two distinct parabolic congruences, there exist $\infty^{2}$ surfaces $S\left(x_{(12)}\right)$, each bearing a family of asymptotic curves in relation $F^{\prime}$ with $S\left(x_{1,1}\right)$ and $S\left(x_{2,2}\right)$. 
By employing the notation used in $\$ \S 4,5$, and 6 , which is similar to that used by Eisenhart, ${ }^{*}$ the equations in these sections can be given metric interpretations which yield a theory of parallel transformations for families of asymptotic curves, and also radial transformations of the same.

7. General transversal surfaces of a parabolic congruence. Consider $S(x)$, the most general transversal surface of a parabolic congruence. Let the curves cut out on $S(x)$ by the developable surfaces of the congruence be used as parametric $u$-curves. It has been demonstrated by the author $\dagger$ that under these conditions the coordinates $x$ satisfy an integrable system of differential equations of the form

$$
\begin{aligned}
x_{u u u} & =a x_{u u}+2 b x_{u v}+c x_{v v}+d x_{u}+e x_{v}+f x, \\
x_{u u v} & =a^{\prime} x_{u u}+2 b^{\prime} x_{u v}+c^{\prime} x_{v v}+d^{\prime} x_{u}+e^{\prime} x_{v}+f^{\prime} x .
\end{aligned}
$$

These equations require the following conditions of integrability:

$$
\begin{aligned}
& c=2 b-c^{\prime}=0, \\
& a c^{\prime}-c_{u}^{\prime}+e-2 b^{\prime} c^{\prime}=0, \\
& 2 b_{v}+2 a b^{\prime}-2 a^{\prime} b+d-2 b_{u}^{\prime}-4 b^{\prime 2}-e^{\prime}=0, \\
& a_{v}-a_{u}^{\prime}-d^{\prime}-2 a^{\prime} b^{\prime}=0, \\
& e_{v}+a e^{\prime}-a^{\prime} e-2 b^{\prime} e^{\prime}-e_{u}^{\prime}+f=0, \\
& d_{v}+a d^{\prime}-a^{\prime} d-2 b^{\prime} d^{\prime}-d_{u}^{\prime}-f^{\prime}=0, \\
& f_{v}+a f^{\prime}-a^{\prime} f-2 b^{\prime} f^{\prime}-f_{u}^{\prime}=0 .
\end{aligned}
$$

In the sense of Definition 1 the $u$-curves of the surface $S(x)$ are autoconjugate to the parabolic congruence to which $S(x)$ is transversal.

To determine the focal surface $S(y)$ of a parabolic congruence which is autoconjugate to the $u$-curves of $S(x)$, an integral surface of (7.1), consider the point having coordinates

$$
y=x_{u u}-2 b^{\prime} x_{u}-c^{\prime} x_{v}+\left(c_{v}^{\prime}-a^{\prime} c^{\prime}-e^{\prime}\right) x .
$$

Using equations (7.1) and (7.2) it can be verified that $x$ and $y$ are related by equations of the form

$$
\begin{aligned}
& y_{u}-\left(a-2 b^{\prime}\right) y=G x, \\
& y_{v}-a^{\prime} y=h x+k x_{u} .
\end{aligned}
$$

On eliminating $x$ from (7.4) it is found that the coordinates $y$ satisfy an equation

* Eisenhart, loc. cit., ch. 2.

† Gore, University of Chicago Dissertation, 1932, p. 47. 


$$
y_{u u}=\alpha y_{u}+\beta y_{v}+\gamma y .
$$

The first of equations (7.4) shows that $S(x)$ is transversal to the $u$-tangent lines of $S(y)$, while equation (7.5) indicates that the $u$-curves of $S(y)$ are a family of asymptotic curves.

Since the surface $S(y)$ can be transformed into a sequence of surfaces by transformations of the type of (1.2) and since the surface $S(x)$ is transversal to the connecting tangent lines between two consecutive surfaces, we can make use of a general inscribing theorem* which we quote:

"Let $T$ denote a sequence of surfaces in which the points" of each surface $\Sigma_{i+1}$ are joined in a one-to-one manner to the corresponding points of $\Sigma_{i}$ by a set $\Omega_{i}$ of $\infty^{2}$ osculating spaces of $\nu$ dimensions belonging to the curves on the surface $\Sigma_{i}$. Let $\Sigma_{r}^{\prime}$ be any surface that is transversal to the set of osculants $\Omega_{r}$. Then it follows that the transversal surface $\Sigma_{r}^{\prime}$ belongs to a sequence of surfaces $T^{\prime}$ which is inscribed in the given sequence T."

The above theorem shows that the surface $S(x)$ belongs to a sequence that is inscribed in the sequence to which $S(y)$ belongs. But since $S(y)$ can be transformed into a multiplicity of sequences, the same is true of $S(x)$. The transformations that operate to produce them are similar to (1.2).

* Gore, Inscribed sequences of surfaces associated with generalized sequences of Laplace, these Transactions, vol. 36 (1934), p. 532.

Central Y.M.C.A. College, Chicago, Ill. 